\title{
Synthesis of Quinolines via Pd/C-Catalyzed Cyclization of 2-Aminobenzyl Alcohol with Ketones
}

\author{
Chan Sik Cho, ${ }^{\dagger}, *$ Wen Xiu Ren, and Sang Chul Shim" \\ ${ }^{\dagger}$ Research Institute of Industrial Technology, Kyungpook National University, Daegu 702-701, Korea. *E-mail: cscho@knu.ac.kr \\ Department of Applied Chemistry, College of Engineering, Kyungpook National University, Daegu 702-701, Korea \\ *E-mail:scshim@knu.ac.kr \\ Received May 27, 2005
}

Key Words : 2-Aminobenzyl alcohol, Ketones, Oxidative cyclization, Pd/C catalyst, Quinolines

It is known that quinoline plays an important role as a basic skeleton for the design of many pharmacologically active compounds such as antiasthmatic, anti-inflammatory and antimalarial. ${ }^{1}$ During the course of our studies directed towards C-N bond activation, ${ }^{2,3}$ we have reported the ruthenium-catalyzed synthesis of quinolines via an alkyl or alkanol group transfer from alkylamines or alkanolamines to $\mathrm{N}$-atom of anilines ${ }^{4}$ (amine exchange reaction ${ }^{5}$ ), followed by cascade isomerization and cyclization of 3-(2-aminophenyl)1-arylprop-2-yn-1-ols. ${ }^{6}$ In connection with this report, several routes for the coupling of carbonyl compounds and alcohols have recently been reported as exemplified in Scheme 1..$^{7-9}$ The coupling of ketones A with primary alcohols $\mathbf{B}$ preferentially afforded the coupled ketones $\mathbf{C}$ (Scheme 1 , route a) ${ }^{7}$ or the coupled secondary alcohols D (Scheme 1, route $b)^{8}$ which depend on the molar ratio of $\mathbf{B}$ to A. In addition, secondary alcohols $\mathbf{E}$ was also found to be coupled with $\mathbf{B}$ to give $\mathbf{D}$ (Scheme 1 , route c). ${ }^{9}$ These reactions could be applied to modified Friedländer quinoline synthesis via ruthenium-catalyzed consecutive coupling and cyclization of 2-aminobenzyl alcohol with ketones and secondary alcohols, ${ }^{10-12}$ which is superior to conventional Friedländer method in a sense of price and stability of 2aminobenzyl alcohol. ${ }^{13}$ Under these circumstances, this report describes an alternative palladium-catalyzed route for Friedländer quinoline synthesis. ${ }^{14,15}$

Table 1 shows several attempted results for the oxidative coupling and cyclization of 2-aminobenzyl alcohol (1) with acetophenone (2a). Generally, treatment of $\mathbf{1}$ with $\mathbf{2 a}$ in dioxane in the presence of a catalytic amount of $5 \% \mathrm{Pd} / \mathrm{C}$ $(0.5 \mathrm{~mol} \%)$ and $\mathrm{KOH}$ at $100{ }^{\circ} \mathrm{C}$ afforded 2-phenylquinoline (3a) with concomitant formation of direct transfer hydrogenation product, 1-phenylethanol (4). The yield of $\mathbf{3 a}$

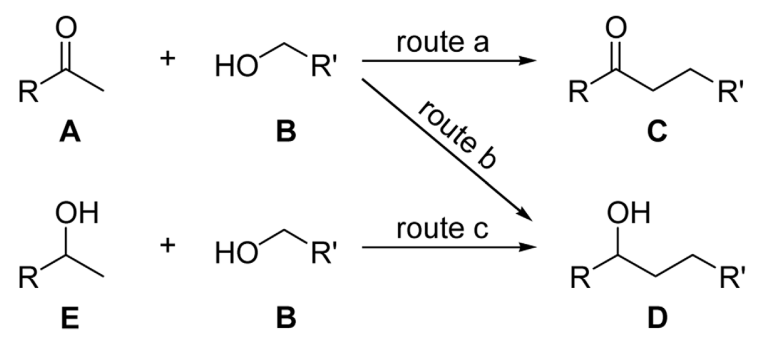

Scheme 1 increased with the reaction time up to $20 \mathrm{~h}$ (runs 1-4) and the amount of $\mathrm{KOH}$ employed (runs 4-6). As has been observed in our recent ruthenium-catalyzed version, ${ }^{10}$ the molar ratio of 2a to 1 also affected the yield of 3a, higher molar ratio up to $[\mathbf{2} \mathbf{a}] /[\mathbf{1}]=2$ resulting in the effective formation of $\mathbf{3 a}$ (runs 4 and 7). This could be due to the acceleration of the initial oxidation of 1 to 2-aminobenzaldehyde by transfer hydrogenation from 1 to excess $\mathbf{2 a} .{ }^{16}$ However, performing the reaction in the presence of 1-decene as a hydrogen acceptor instead of excess $\mathbf{2 a}$ gave no significant change on the product yield and distribution (run 8).

Having established reaction conditions, various ketones 2 were subjected to react with $\mathbf{1}$ in order to investigate the reaction scope and several representative results are summarized in Table 2. From the reactions between $\mathbf{1}$ and aryl(methyl) ketones (2a-2h), the corresponding 2-arylquinolines (3a-3h) were produced in the range of $43-77 \%$ yields. Here again, the conventional transfer hydrogenated aryl(methyl) carbinols were produced in considerable amounts on GLC analysis. The position and electronic nature of the substituent on the aromatic ring of aryl(metnyl) ketones had no relevance to quinoline yield. The reaction proceeds likewise with heteroaryl(methyl) ketone $\mathbf{2 i}$ and 2'-

Table 1. Pd/C-catalyzed optimization of conditions for the reaction of $\mathbf{1}$ with $\mathbf{2} \mathbf{a}^{a}$

\begin{tabular}{|c|c|c|c|c|c|c|}
\hline \multirow{2}{*}{ Run } & \multirow{2}{*}{$2 a] /[1]$} & \multirow{2}{*}{$\begin{array}{l}\mathrm{KOH} \\
(\mathrm{mmol})\end{array}$} & \multirow{2}{*}{ Time (h) } & \multirow{2}{*}{$\begin{array}{c}\text { Conv. }(\%) \\
\text { of } \mathbf{2 a}\end{array}$} & \multicolumn{2}{|c|}{ GLC yield $(\%)$} \\
\hline & & & & & $3 \mathbf{a}$ & 4 \\
\hline 1 & 2 & 2 & 5 & 63 & 54 & 32 \\
\hline 2 & 2 & 2 & 10 & 76 & 67 & 44 \\
\hline 3 & 2 & 2 & 15 & 87 & 76 & 43 \\
\hline 4 & 2 & 2 & 20 & 88 & 87 & 53 \\
\hline 5 & 2 & 1 & 20 & 75 & 70 & 45 \\
\hline 6 & 2 & 3 & 20 & 90 & 86 & 52 \\
\hline 7 & 1 & 2 & 20 & 100 & 60 & 28 \\
\hline $8^{c}$ & 1 & 2 & 20 & 100 & 56 & 28 \\
\hline
\end{tabular}

${ }^{a}$ Reaction conditions: 1 ( $\left.1 \mathrm{mmol}\right), \mathrm{Pd} / \mathrm{C}(0.005 \mathrm{mmol})$, dioxane $(3 \mathrm{~mL})$, $100{ }^{\circ} \mathrm{C} .{ }^{b}$ Based on $1 .{ }^{c}$ In the presence of 1 -decene $(2 \mathrm{mmol})$. 
Table 2. Pd/C-catalyzed synthesis of quinolines $\mathbf{3}$ from $\mathbf{1}$ and $\mathbf{2}^{a}$

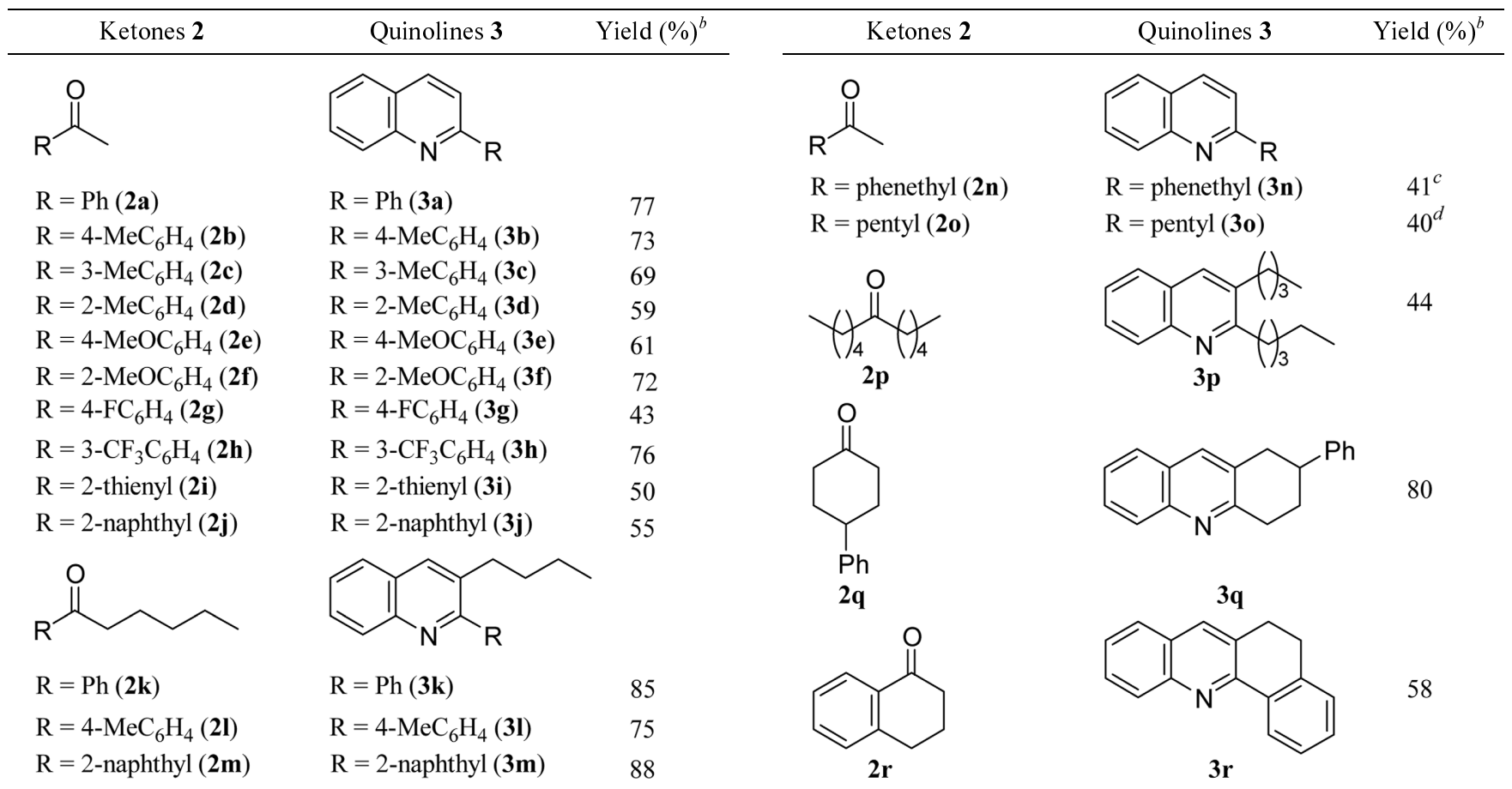

${ }^{a}$ Reaction conditions: $1(1 \mathrm{mmol}), 2(2 \mathrm{mmol}), \mathrm{Pd} / \mathrm{C}(0.005 \mathrm{mmol}), \mathrm{KOH}(2 \mathrm{mmol})$, dioxane $(3 \mathrm{~mL}), 100{ }^{\circ} \mathrm{C}, 20 \mathrm{~h} .{ }^{b}$ Isolated yield. ${ }^{c} 3-\mathrm{Benzyl}-2-$ methylquinoline was also formed in $20 \%$ yield. ${ }^{3} 3$-Butyl-2-methylquinoline was also formed in $13 \%$ yield.

acetonaphthone $(\mathbf{2} \mathbf{j})$ to give the corresponding quinolines $\mathbf{3 i}$ and $\mathbf{3 j}$. Higher reaction rate and yield were observed with alkyl(aryl) ketones $(\mathbf{2} \mathbf{k}-\mathbf{2 m})$. In the reaction of alkyl(methyl) ketones (2n and 2o) the corresponding quinolines were obtained as a regioisomeric mixture, favoring cyclization at less-hindered position over $\alpha$-methylene..$^{2 \mathrm{a}, 7-10,17}$ With dialkyl ketone 2p, 3-butyl-2-pentylquinoline (3p) was also formed in $44 \%$ yield. Cyclic ketones such as 4-phenylcyclohexanone $(\mathbf{2 q})$ and 1-tetralone (2r) were also reacted with $\mathbf{1}$ to give 3-phenyl-1,2,3,4-tetrahydroacridine (3q) and 5,6-dihydrobenzo $[c]$ acridine (3r) in $80 \%$ and $58 \%$ yields, respectively.

The catalytic pathway seems to proceed via initial oxidation of 1 to 2-aminobenzaldehyde (5), which in turn triggers cross aldol condensation with 2a under $\mathrm{KOH}$ to give an $\alpha, \beta$-unsaturated ketone $\mathbf{6}$. This is followed by cyclodehydration to form 3a (Scheme 2). ${ }^{10}$ An alternative route for 3a involves a sequence such as ketimine 7 formation from 1 and $\mathbf{2 a}$, oxidative addition of palladium to $\mathrm{O}-\mathrm{H}$ bond of $\mathbf{7}$ to give palladium hydride complex $\mathbf{8}, \beta$-hydride elimination from $\mathbf{8}$ to form ketimine aldehyde $\mathbf{9}$ and intramolecular aldol-type condensation of $9 .{ }^{12}$

In summary, it has been shown that 2-aminobenzyl alcohol undergoes an oxidative coupling and cyclization with ketones in the presence of a catalytic amount of $\mathrm{Pd} / \mathrm{C}$ along with $\mathrm{KOH}$ to give quinolines in moderate to good yields. To best of our knowledge, the present reaction is the<smiles>Nc1ccccc1CO</smiles>

1<smiles>CC(O)c1ccccc1</smiles>

4<smiles>C/C(=N\c1ccccc1CO)c1ccccc1</smiles>

7

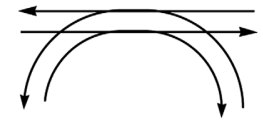

$[\mathrm{Pd}]$

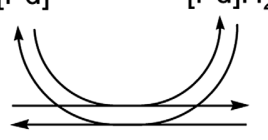<smiles>C/C(=N/c1ccccc1COP)c1ccccc1</smiles>

8

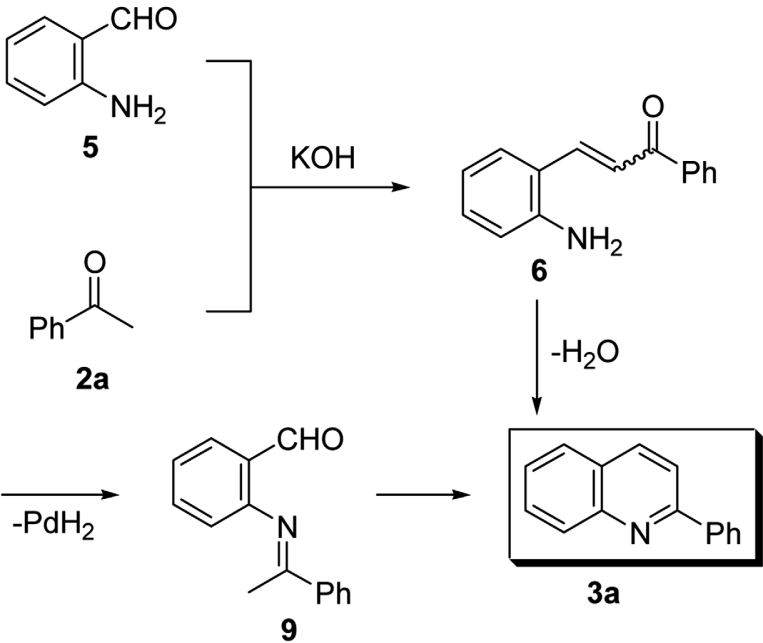

Scheme 2 
first example for palladium-catalyzed Friedländer quinoline synthesis.

\section{Experimental Section}

${ }^{1} \mathrm{H}$ and ${ }^{13} \mathrm{C}$ NMR (400 and $100 \mathrm{MHz}$ ) spectra were recorded on a Bruker Avance Digital 400 spectrometer using TMS as an internal standard. GLC analyses were carried out with a Shimadzu GC-17A instrument equipped with a CBP10-S25-050 column (Shimadzu, fused silica capillary column, $0.33 \mathrm{~mm} \times 25 \mathrm{~m}, 0.25 \mu \mathrm{m}$ film thickness) using nitrogen as carrier gas. The isolation of pure products was carried out via thin layer chromatography (silica gel 60 $\mathrm{GF}_{254}$, Merck). Commercially available organic and inorganic compounds were used without further purification.

General experimental procedure. A mixture of 2aminobenzyl alcohol $(0.123 \mathrm{~g}, 1 \mathrm{mmol})$, ketone (2 mmol), palladium, 5 wt.\% on activated carbon $(0.011 \mathrm{~g}, 0.005$ $\mathrm{mmol})$ and $\mathrm{KOH}(0.112 \mathrm{~g}, 2 \mathrm{mmol})$ in dioxane $(3 \mathrm{~mL})$ was placed in a $5 \mathrm{~mL}$ screw-capped vial and allowed to react at $100{ }^{\circ} \mathrm{C}$ for $20 \mathrm{~h}$. The reaction mixture was filtered through a short silica gel column (ethyl acetate-hexane mixture) to eliminate inorganic salts. To the extract was added appropriate amount of undecane as an internal standard and analyzed by GLC for the determination of the conversion of 2a and the yield of 3a and 4. Removal of the solvent left a crude mixture, which was separated by thin layer chromatography (silica gel, ethyl acetate-hexane mixture) to give quinolines 3. All products prepared by the above procedure were identified by comparison with samples noted in our recent report except for $\mathbf{3 k - 3}$.

3-Butyl-2-phenylquinoline (3k). Pale yellow oil; ${ }^{1} \mathrm{H}$ $\operatorname{NMR}\left(\mathrm{CDCl}_{3}\right) \delta 0.80(\mathrm{t}, J=7.3 \mathrm{~Hz}, 3 \mathrm{H}), 1.19-1.28(\mathrm{~m}, 2 \mathrm{H})$, $1.45-1.53(\mathrm{~m}, 2 \mathrm{H}), 2.74(\mathrm{t}, J=8.0 \mathrm{~Hz}, 2 \mathrm{H}), 7.38-7.49(\mathrm{~m}$, 4H), $7.54(\mathrm{~d}, J=7.0 \mathrm{~Hz}, 2 \mathrm{H}), 7.63(\mathrm{t}, J=7.5 \mathrm{~Hz}, 1 \mathrm{H}), 7.75$ $(\mathrm{d}, J=8.0 \mathrm{~Hz}, 1 \mathrm{H}), 7.98(\mathrm{~s}, 1 \mathrm{H}), 8.14(\mathrm{~d}, J=8.6 \mathrm{~Hz}, 1 \mathrm{H})$; ${ }^{13} \mathrm{C} \mathrm{NMR}\left(\mathrm{CDCl}_{3}\right) \delta 14.5,23.0,33.1,33.4,127.0,127.6$, 128.3, 128.7, 128.9, $129.4(\times 2), 129.9,134.7,136.3,141.6$, 147.0, 161.4. Anal. Calcd for $\mathrm{C}_{19} \mathrm{H}_{19} \mathrm{~N}: \mathrm{C}, 87.31 ; \mathrm{H}, 7.33 ; \mathrm{N}$, 5.36. Found: C, 87.29; H, 7.69; N, 5.42.

3-Butyl-2-(4-methylphenyl)quinoline (3I). Pale yellow oil; ${ }^{1} \mathrm{H}$ NMR $\left(\mathrm{CDCl}_{3}\right) \delta 0.83(\mathrm{t}, J=7.3 \mathrm{~Hz}, 3 \mathrm{H}), 1.22-1.31$ $(\mathrm{m}, 2 \mathrm{H}), 1.48-1.56(\mathrm{~m}, 2 \mathrm{H}), 2.41(\mathrm{~s}, 3 \mathrm{H}), 2.77(\mathrm{t}, J=8.0 \mathrm{~Hz}$, 2H), 7.27 (d, $J=7.5 \mathrm{~Hz}, 2 \mathrm{H}), 7.44$ (d, $J=8.0 \mathrm{~Hz}, 2 \mathrm{H}), 7.48$ $(\mathrm{t}, J=7.8 \mathrm{~Hz}, 1 \mathrm{H}), 7.63(\mathrm{t}, J=7.3 \mathrm{~Hz}, 1 \mathrm{H}), 7.76 \mathrm{~d}, J=7.5$ $\mathrm{Hz}, 1 \mathrm{H}), 8.00(\mathrm{~s}, 1 \mathrm{H}), 8.12(\mathrm{~d}, J=8.5 \mathrm{~Hz}, 1 \mathrm{H}) ;{ }^{13} \mathrm{C} \mathrm{NMR}$ $\left(\mathrm{CDCl}_{3}\right) \delta 14.3,21.8,22.8,33.0,33.2,126.6,127.3,128.0$, 129.1 ( $\times 2), 129.4,129.7,134.6,136.0,138.2,138.5,146.8$, 161.2. Anal. Calcd for $\mathrm{C}_{20} \mathrm{H}_{21} \mathrm{~N}$ : C, 87.23; H, 7.69; N, 5.09. Found: C, 87.25; H, 8.04; N, 5.13.

3-Butyl-2-(2-naphthyl)quinoline (3m). ${ }^{18}$ Pale yellow oil; ${ }^{1} \mathrm{H}$ NMR $\left(\mathrm{CDCl}_{3}\right) \delta 0.76(\mathrm{t}, J=7.3 \mathrm{~Hz}, 3 \mathrm{H}), 1.16-1.25(\mathrm{~m}$, $2 \mathrm{H}), 1.47-1.55(\mathrm{~m}, 2 \mathrm{H}), 2.78(\mathrm{t}, J=7.8 \mathrm{~Hz}, 2 \mathrm{H}), 7.45-7.49$ $(\mathrm{m}, 3 \mathrm{H}), 7.62-7.69(\mathrm{~m}, 2 \mathrm{H}), 7.76(\mathrm{~d}, J=8.0 \mathrm{~Hz}, 1 \mathrm{H}), 7.85-$ $7.89(\mathrm{~m}, 2 \mathrm{H}), 7.92(\mathrm{~d}, J=8.5 \mathrm{~Hz}, 1 \mathrm{H}), 8.02(\mathrm{~d}, J=8.5 \mathrm{~Hz}$, $2 \mathrm{H}), 8.18(\mathrm{~d}, J=8.5 \mathrm{~Hz}, 1 \mathrm{H}) ;{ }^{13} \mathrm{C} \mathrm{NMR}\left(\mathrm{CDCl}_{3}\right) \delta 14.3$, $22.9,33.0,33.3,126.7,126.8,126.9,127.2,127.5,128.1$,
$128.2,128.4,128.6,128.9,129.4,129.8,133.5,133.7$, $134.7,136.3,138.9,146.9,161.1$.

Acknowledgment. The present work was supported by BK-21 in 2003 and a Research Foundation Grant (KRF2002-070-C00055). C.S.C. gratefully acknowledges a Research Professor Grant of Kyungpook National University (2004).

\section{References}

1. (a) Jones, G. Comprehensive Heterocyclic Chemistry; Katritzky, A. R., Rees, C. W., Eds.; Pergamon: New York, 1984; Vol. 2, p 395. (b) Arcadi, A.; Chiarini, M.; Giuseppe, S. D.; Marinelli, F. Synlett 2003, 203, and references cited therein. (c) Cho, S. Y.; Ahn, J. H.; Ha, J. D.; Kang, S. K.; Baek, J. Y.; Han, S. S.; Shin, E. Y.; Kim, S. S.; Kim, K. R.; Cheon, H. G.; Choi, J.-K. Bull. Korean Chem. Soc. 2003, 24, 1455.

2. (a) Cho, C. S.; Kim, B. T.; Lee, M. J.; Kim, T.-J.; Shim, S. C. Angew. Chem., Int. Ed. 2001, 40, 958. (b) Cho, C. S.; Park, J. H.; Kim, T.-J.; Shim, S. C. Bull. Korean Chem. Soc. 2002, 23, 23.

3. Cho, C. S. Tetrahedron Lett. 2005, 46, 1415.

4. (a) Cho, C. S.; Oh, B. H.; Shim, S. C. Tetrahedron Lett. 1999, 40, 1499. (b) Cho, C. S.; Oh, B. H.; Shim, S. C. J. Heterocycl. Chem. 1999, 36, 1175. (c) Cho, C. S.; Kim, J. S.; Oh, B. H.; Kim, T.-J.; Shim, S. C. Tetrahedron 2000, 56, 7747. (d) Cho, C. S.; Oh, B. H.; Kim, J. S.; Kim, T.-J.; Shim, S. C. Chem. Commun. 2000, 1885. (e) Cho, C. S.; Kim, T. K.; Kim, B. T.; Kim, T.-J.; Shim, S. C. J. Organomet. Chem. 2002, 650, 65. (f) Cho, C. S.; Lee, N. Y.; Kim, T.-J.; Shim, S. C. J. Heterocyclic Chem. 2004, 41, 423.

5. Murahashi, S.-I. Angew. Chem., Int. Ed. 1995, 34, 2443.

6. Cho, C. S.; Lee, N. Y.; Kim, T.-J.; Shim, S. C. J. Heterocyclic Chem. 2004, 41, 409.

7. Cho, C. S.; Kim, B. T.; Kim, T.-J.; Shim, S. C. Tetrahedron Lett. 2002, 43, 7987.

8. Cho, C. S.; Kim, B. T.; Kim, T.-J.; Shim, S. C. J. Org. Chem. 2001, 66, 9022 .

9. Cho, C. S.; Kim, B. T.; Kim, H.-S.; Kim, T.-J.; Shim, S. C. Organometallics 2003, 22, 3608.

10. (a) Cho, C. S.; Kim, B. T.; Kim, T.-J.; Shim, S. C. Chem. Commun. 2001, 2576. (b) Cho, C. S.; Kim, B. T.; Choi, H.-J.; Kim, T.-J.; Shim, S. C. Tetrahedron 2003, 59, 7997.

11. Motokura, K.; Mizugaki, T.; Ebitani, K.; Kaneda, K. Tetrahedron Lett. 2004, 45, 6029.

12. For iridium-catalyzed cyclization of 2-aminobenzyl alcohol with ketones leading to quinolines: Taguchi, K.; Sakaguchi, S.; Ishii, Y. Tetrahedron Lett. 2005, 46, 4539.

13. (a) Friedläender, P. Chem. Ber. 1882, 15, 2572. (b) For a review, see: Cheng, C.-C.; Yan, S.-J. Org. Reactions 1982, $28,37$.

14. For our recent report on palladium catalysis: (a) Cho, C. S.; Lim, D. K.; Heo, N. H.; Kim, T.-J.; Shim, S. C. Chem. Commun. 2004, 104. (b) Cho, C. S.; Lim, D. K.; Zhang, J. Q.; Kim, T.-J.; Shim, S. C. Tetrahedron Lett. 2004, 45, 5653.

15. Comparing with ruthenium-catalyzed reaction, $\mathrm{Pd} / \mathrm{C}$ can be easily removed after reaction.

16. For recent reviews on transition metal-catalyzed transfer hydrogenation, see: (a) Zassinovich, G.; Mestroni, G.; Gladiali, S. Chem. Rev. 1992, 92, 1051. (b) Bäckvall, J.-E.; Chowdhury, R. L.; Karlsson, U.; Wang, G. Perspectives in Coordination Chemistry; Williams, A. F.; Floriani, C.; Merbach, A. E., Eds.; VCH: New York, 1992; pp 463-486. (c) Noyori, R.; Hashiguchi, S. Acc. Chem. Res. 1997, 30, 97. (d) Naota, T.; Takaya, H.; Murahashi, S.I. Chem. Rev. 1998, 98, 2599. (e) Palmer, M.; Wills, M. Tetrahedron: Asymmetry 1999, 10, 2045.

17. Hsiao, Y.; Rivera, N. R.; Yasuda, N.; Hughes, D. L.; Reider, P. J. Org. Lett. 2001, 3, 1101.

18. Garrett, R. D.; Henze, H. R. J. Med. Chem. 1966, 9, 976. 